\title{
Health Care Workers Humoral Immune Response Against GLcB, MPT51 and HSPX from Mycobacterium tuberculosis
}

\author{
Michelle Cristina Guerreiro dos Reis, Marcelo Fouad Rabahi, André Kipnis and Ana Paula Junqueira-Kipnis \\ Federal University of Goiás; Goiânia, GO, Brazil
}

\begin{abstract}
Tuberculosis (TB) is one of the oldest human infectious diseases and one third of the world's population is latently infected. Brazil is an endemic area for TB. One of the most important challenges in TB control is the identification of latently infected individuals. Health Care Workers (HCW) are at high risk of being infected with Mycobacterium tuberculosis and even to become TB latently infected. The aim of this study was to increase knowledge about humoral immune response in TB latently infected individuals. HCW were classified according to their tuberculin skin test (TST), as positive or negative. The antibody response to GLcB, MPT51 and HSPX from Mycobacterium tuberculosis was evaluated. TST negative HCW constituted the majority of those who showed a humoral immune response. Antibody levels varied according to antigen characteristics, TST and BCG status. We suggest that possibly the presence of those antibodies could have a function in the protective immune response against Mycobacterium tuberculosis.
\end{abstract}

Key-Words: Latent tuberculosis, infection, antibodies.

Since antiquity, tuberculosis (TB) has remained a serious public health problem, claiming the lives of 1,700,000 people in 2004 [1]. It is caused by intracellular bacilli Mycobacterium tuberculosis and lungs are most frequently affected organs [2].

In vitro cultures of M.tuberculosis, which mimics the environment found by this agent in human host, shows that these bacilli produce and release many proteins into the extracellular environment [3]. Some of these proteins have been identified and tested for their ability to induce an immune response in mice and humans [4,5]. GLcB (malate synthase), MPT51 (FbpC1) and HSPX (alpha crystalline) are some of the proteins produced by M.tuberculosis in such conditions.

GLcB is an enzyme that catalyses carbon assimilation by the glyoxylate shunt pathway [6] and seems to be involved in M.tuberculosis virulence [7]. MPT51 is a non-catalytic $\alpha / \beta$ hydrolase that may be involved in M.tuberculosis adhesion mechanisms [8]. HSPX is a chaperone involved in maintaining the integrity of bacilli cell wall [9]. These antigens were chosen for this study, because these proteins are expressed in the early stages of infection [3], are recognized by both healthy infected individuals and patients with active tuberculosis $[10,11]$, and their possible use in immune diagnosis have been studied [12-15].

Nonetheless, only $5 \%$ of infected individuals become ill, the other $95 \%$ of infected individuals remain asymptomatic. TB latently infected individuals are those who were exposed, respond to PPD (purified protein derivative, used in the tuberculosis skin test) and do not present any symptoms of Received on 15 May 2009; revised 16 November 2009.

Address for correspondence: Dr. Ana Paula Junqueira-Kipnis. Instituto de Patologia Tropical e Saúde Pública. Laboratório de Imunopatologia das Doenças Infecciosas. Rua 235 esquina com Primeira Avenida, S/N. Setor Universitario. Zip code: 74605-050. Phone: 5562 32096126, Fax: 55 62 32096363. Goiania, Goias, Brazil. This study was supported by CNPq and FUNAPE (Brazil).

The Brazilian Journal of Infectious Diseases 2009;13(6):417-421. (C) 2009 by The Brazilian Journal of Infectious Diseases and Contexto Publishing. All rights reserved. the disease. Health care workers (HCW) are more frequently exposed to infectious agents, such as M.tuberculosis, than general population, so they are more likely to become ТB latently infected individuals [16].

The TST positivity is high among HCW in developing countries [17-19] and this correlate with high prevalence of latently infected individuals and high risk of getting TB in such countries [20]. However, the majority of the HCW, despite their latent infection, do not become sick [17, 21]. Knowledge of their humoral immune response is limited. Several mycobacterial antigens are recognized by human antibodies $[5,12,13,15,22]$. Some studies have also shown that there is an effective role for antibodies against intracellular pathogens [23,24].

The aim of this study was to increase knowledge of humoral immune response in latently infected individuals. We analyzed antibody response of TB latently infected HCW against antigens GLcB, HSPX and MPT51. We showed that the majority of the HCW present antibodies (IgM/IgG) against at least one of the three tested antigens.

\section{Material and Methods}

Health Care Workers

Individuals were recruited from Anuar Auad Hospital (Anuar Auad Tropical Disease Hospital, HDTAA) in Goiânia, Goiás, Brazil, between June 2001 until June 2004. HDTAA is one of the TB treatment reference center in Goiás. The inclusion criteria were as follows: work schedule of at least 20 hours/week, age greater than 18 years, any sex or race, free from chronic disease or pregnancy and a negative HIV test. Serum samples were taken from 299 HCW from all clinical settings, including ambulatory, administration, nursery, surgery units, laboratories, laundry, nutrition and pharmacy. Samples were stored at $-20^{\circ} \mathrm{C}$ until further use. All participants were classified by TST (2 UI - PPDRT23, Statens Serum Institute, Copenhagen) as positive $(\geq 10 \mathrm{~mm})$ or negative $(<9 \mathrm{~mm})$. Individuals who had positive TST were considered in this study as TB latently infected. In the cohort of 299 
HCW, 122 were TST positive and 177 were TST negative. BCG status was certificated by the presence of a vaccinal scar on the upper right arm. Most of the participants were women presenting an average age of 48 years, were BCG vaccinated and they had been working in public health for at least 11 years. Socio-demographic characteristics of the HCW cohort are shown in Table 1. The HDTAA Ethics Committee approved this study and all individuals provided written and informed consent prior to their participation. All individuals were healthy at the time of the study.

Mycobacterium tuberculosis Antigens (GLcB, MPT51 and HSPX)

Recombinant protein antigens were produced by the Colorado State University and provided to Universidade Federal de Goiás by a MTA- NO1-Al - 75320. The recombinant proteins were produced according to previous studies $[3,6,25,26]$.

Enzyme-Linked Immunosabsorbent Assay (ELISA)

Recombinant antigen $(50 \mu \mathrm{L}, 2.5 \mu \mathrm{g} / \mathrm{mL}$, in sodium carbonate pH 9.6) was used to coat the ELISA plates (NUNC). The plates were blocked with PBS/1\% fat-free milk for 2 hours at $37^{\circ} \mathrm{C}$. Sera were diluted 1:200 for IgM and 1:400 for IgG in PBS/0.05\% fat-free milk, and $50 \mu \mathrm{L}$ of the dilution was added to each well and incubated at $37^{\circ} \mathrm{C}$ for 2 hours. Anti-human immunoglobulin against both IgM and IgG conjugated with peroxidase (Bio-Rad) was diluted 1:3000 for IgM and 1:15000 for IgG in PBS/0.05\% fat-free milk. Fifty $\mu \mathrm{L}$ of these dilutions were added to each well and then incubated for 1 hour at $37^{\circ} \mathrm{C}$. Fifty $\mu \mathrm{L}$ of developing solution $(5 \mathrm{mg} / \mathrm{mL}$ ortho phenyl toluidine $+\mathrm{H}_{2} \mathrm{O}_{2}+$ citrate-phosphate buffer ph 5.0) was added to each well and left in the dark for 15 minutes. After stopping the reaction with $50 \mu \mathrm{L}$ of $4 \mathrm{~N} \mathrm{H}_{2} \mathrm{SO}_{4}$, the plates were read at 492 nm using a Thermo Systems Multiskan instrument. Between all incubations, the plates were washed six times with PBS/0.05\% Tween 20.

The cut off was determined as follows: sera from eight healthy individuals with negative TST, who were not HCW, and to their knowledge never contacted TB or leprosy patients was used as a negative control, and the results of the optical density for each antigen and immunoglobulin were determined. The cut off was determined as the average of the optical densities to all tested antigen plus two standards deviation. Thus for IgM, optical densities above 0.300 , and for IgG optical densities above 0.350 were considered positive.

Statistical Analyses

ANOVA and Student's $t$ test were used to analyze the results. Significance was defined as $\mathrm{p}<0.05$.

\section{Results}

Health care workers (HCW) presented antibody response to M.tuberculosis antigens. Essentially 99\% of HCW (296/ 299) had IgM antibodies against GLcB, of those 59.5\% were
TST-negative. A slightly lower proportion of HCW (90\%, 269/ 299) presented IgG antibodies against GLcB, of those $58 \%$ were TST-negative. The levels of both IgM and IgG antibodies against GLcB were similar between TST-negative and positive HCW (Figures 1a and 1b).

Regarding HSPX, 95\% of HCW (285/299) presented IgM antibodies against it, of these 59\% were TST-negative. TSTnegative HCW showed slightly higher levels of IgM than TST-positive HCW ( $<$ 0.05) (Figure 1c). When IgG against HSPX was evaluated, only $10 \%$ of HCW showed antibodies (30/299). Of those 57\% were TST-negative and 43\% TSTpositive. Levels of IgG against HSPX were similar between TST-negative and TST-positive HCW (Figure 1d).

Only one of HCW (1/299) presented IgM antibodies against MPT51 and this individual was TST-negative. One hundred and forty six HCW (49\%) had IgG antibodies against MPT51, of which 61\% were TST-negative (Figures 1e and 1f). Levels of IgM and IgG were different for HSPX dosages, in this case IgG levels were higher than IgM.

About 77\% of HCW were BCG-vaccinated (199/299). To determine whether BCG vaccination affected antibody's production, antibody levels were compared between BCGvaccinated and BCG non-vaccinated HCW. In spite of antibody's class, or antigen, antibody levels were similar between those two groups. Table 2 shows the means and standard deviations (sd) of antibody's levels against GLcB, HSPX and MPT51.

\section{Discussion}

In this study most of the HCW showed antibodies (IgM/ IgG) against at least one of the three tested antigens. The dominance of individuals presenting antibodies was among TST-negative ones. Antibody levels were similar between TST positive and negative HCW for IgM against GLcB, and also similar for IgG recognizing GLcB, HSPX and MPT51. Regarding IgM against HSPX, TST negative HCW had higher antibody levels than positive TST HCW. Only one negative TST HCW presented IgM antibodies against MPT51. Antibody's levels were also similar between BCG vaccinated and BCG nonvaccinated individuals.

All participants were classified according to their TST status. Tuberculin skin test is used for more than a century, and despite its limitations [27], still is a useful tool to identify TB latently infected individuals. Surprisingly, HCW who did not respond to PPD, and consequently were not infected, showed antibodies against M.tuberculosis antigens. This contradiction maybe due to the fact that some individuals may not react to PPD [28] or this 'false' negative TST may be due some immunosuppressive conditions [29].

It is expected that in a TB endemic area, like Brazil, several individuals would be TST positive, as we found here in our study. Farhat and colleges [30] showed that BCG has little influence on TST status (in adults persons, for instance), and non-tuberculous mycobacteria are not an important cause of false-positive TST. 
Table 1. Socio-demographic characteristics of the cohort of HCW from HDTAA Goiânia-Brazil examined in this study.

\begin{tabular}{lcccc}
\hline & Participants (n=299) & Age (average- years) & $\begin{array}{c}\text { BCG status } \\
\text { (vaccinated/total\%) }\end{array}$ & $\begin{array}{c}\text { Time of work in health care } \\
\text { system (average years) }\end{array}$ \\
\hline Men & 41 & 38.29 & $30 / 41$ & $(73 \%) 7.65$ \\
Women & 258 & 48.46 & $169 / 258$ & $(65.5 \%) 11.34$ \\
\hline
\end{tabular}

Table 2. Immunoglobulin levels (IgM/ IgG) against recombinant antigens from M.tb in BCG vaccinated and BCG non-vaccinated HCW.

\begin{tabular}{lcccccc}
\hline & $\begin{array}{c}\text { GLcB } \\
\text { IgM }\end{array}$ & $\begin{array}{c}\text { HSPX } \\
\text { IgG }\end{array}$ & $\begin{array}{c}\text { MPT51 } \\
\text { IgM }\end{array}$ & IgG & IgM & IgG \\
\hline BCG - vaccinated & $0.923 \pm 0.3$ & $1.080 \pm 0.6$ & $1.075 \pm 0.4$ & $0.361 \pm 0.2$ & - & $0.447 \pm 0.2$ \\
Non-BCG vaccinated & $0.983 \pm 0.2$ & $1.024 \pm 0.5$ & $1.106 \pm 0.5$ & $0.339 \pm 0.1$ & - & $0.465 \pm 0.2$ \\
\hline
\end{tabular}

The results are shown as average and standard deviations of ELISA optical densities.

Figure 1. Levels of immunoglobulin (IgM/IgG) against recombinant GLcB, HSPX and MPT51 from M.tb in 299 HCW from HDTAA of Goiânia. HCW were grouped according to their TST results, which 177 were TST negative and 122 TST positive. The continuous line represents optic density's means and broken line the cut off. Antibodies levels were similar between negative and positive TST HCW for all dosages, except for HSPX/IgM $(\mathrm{p}<0.05)$, where negative TST HCW showed higher antibody's level than positive TST HCW.
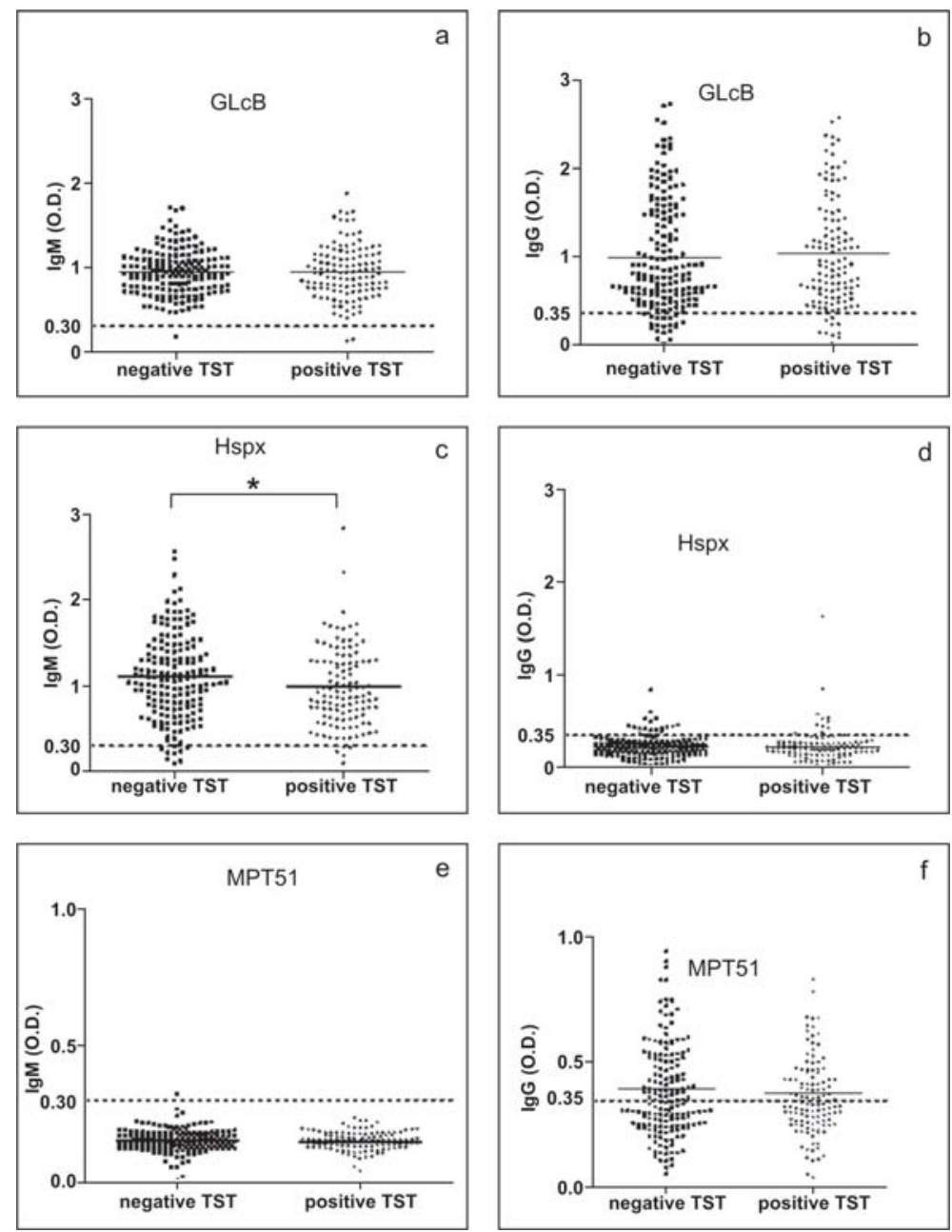

www.bjid.com.br 
The majority of individuals that presented humoral immune response against the mycobacterial antigens used in our study were TST negative. The influence of non-tuberculous mycobacteria on humoral immune response cannot be excluded [31]. In fact, studies have shown that healthy individuals do present antibodies against non-tuberculous mycobacteria that may cross react with some M.tuberculosis antigens [32-34]. Additionally, there is a great difference between the immunological principles of the above-mentioned techniques where TST is based on a cellular response and antibody is based on a humoral response.

Samanich et al. [34] found that tuberculosis patients smear positive or negative, with cavitary or noncavitary disease and healthy controls showed a homogenous antibody response against some mycobacterial antigens (including GLcB and MPT51). Lyashchenko et al. showed that tuberculosis patients presented different levels of antibody response against some recombinant M.tuberculosis antigens [13]. The difference between these two studies is that in the first one, sera from all subjects was pre-absorbed with E.coli lysates to reduce crossreactivity with non-tuberculous mycobacteria antigens. In our study we used recombinant antigens, but like Samanich et al. study, we obtained an antibody response despite the individuals (HCW) were not sick.

Malate synthase, GLcB, initially was characterized as an important enzyme of the glyoxylate shunt involved in carbon assimilation as energy source to bacilli [6]. Later it was described that GLcB is also an anchorless adhesin that may contribute to bacilli virulence [7].

Antibody immune response to GLcB has been associated to tuberculosis patients [12,15,34]. It was also shown that this protein could be a marker for incipient subclinical tuberculosis in $\mathrm{HIV}^{+} \mathrm{TB}$ - individuals, which are at high-risk for TB [35]. Like GLcB, MPT51 is also an adhesin but its binding is associated to fibronectin and small sugars [8]. Likewise GLcB, MPT51 also has been associated with antibody immune responses in tuberculosis patients $[11,13,15,34,36]$. HSPX is a heat shock protein produced by bacilli at early stages of infection [9]. HSPX have been related to distinguishing TB patients from healthy latently infected individuals [37] and previous from recent TB latently infected individuals [10]. The roles played by GLcB, HSPX and MPT51, helping M.tuberculosis survival when active disease is developing or ongoing, can explain the presence of antibodies against it in TB patients, however, we showed in this study that antibodies against GLcB, HSPX and MPT51 were also present in healthy HCW. Therefore, it is hard to justify the anti-antigen presence in negative TST HCW. The shadow of non-tuberculous mycobacteria cross reaction rise again.

BCG vaccinated and non-vaccinated HCW showed similar levels of IgM and IgG against GLcB, HSPX and MPT51. Our HCW were vaccinated at birth so seems improbable that BCG has some influence on antibody production in these HCW.
Ota and colleagues showed that BCG vaccination affects antibody level most in newborns [41]. Nabeshima and colleagues showed that previous vaccination with BCG had no significant effect on antibody levels [42]. Moreover, Drowart and colleagues analyzing the effect of BCG on antibody production showed that IgG level was increased only when P64 and not P32 was used as purified antigen, suggesting that individual characteristics of each antigen may have a role too [43].

\section{Conclusion}

In this study, despite inter individual differences, nearly $50 \%$ of participants possessed IgM or IgG antibodies against GLcB, MPT51 and HSPX from $M$. tuberculosis. TST negative HCW constituted the majority of those who showed a humoral immune response. We suggest that maybe those antibodies could play some role in immune response against M.tuberculosis, thus HCW maintain healthy on hostile environment.

\section{Acknowledgements}

We acknowledge Renner Caetano dos Santos, for helping with blood harvest and TST testing.

\section{References}

1. Dye, Christopher. Global Epidemiology of tuberculosis. Lancet, 2006, Vol.367: 938-40.

2. Marais BJ, Obihara CC, Gie RP et al. The prevalence of symptoms associated with pulmonary tuberculosis in randomily selected children from a high burden community. Arch Dis Child, 2005, Vol. 90: 1166-70.

3. Sonnenberg MG, Belisle JT. Definition of Mycobacterium tuberculosis culture filtrate proteins by two-dimensional polyacrilamide gel electrophoresis, N-terminal amino acid sequancing, and electrospray mass spectrometry. Infect Immun, 1997, Vol.65(11): 4515-24.

4. Andersen, P. Effective vaccination of mice against Mycobacterium tuberculosis infection with a soluble mixture of secreted mycobacterial proteins. Infect Immun, 1994, Vol. 62(6): 253644.

5. Havlir DV, Wallis Boom WH, Daniel TM, et al. Human Immune Response to Mycobacterium tuberculosis Antigens. Infect Immun, 1991, Vol. 59(2): 665-670

6. Smith CV, Huang CC, Miczack A, et al. Biochemical and structural studies of malate synthase from Mycobacterium tuberculosis. J Biol Chem, 2003, Vol. 278(3): 1735-43.

7. Kinhikar AG, Vargas D, Li H, et al. Mycobacterium tuberculosis malate synthase is a laminin-binding adhesin. Mol Microbiol, 2006, Vol. 60(4): 999-1013.

8. Wilson RA, Maughan WN, Kremer L, Bestra GS, Futter K. The structure of Mycobacterium tuberculosis MPT51 (FbpC1) defines a new family of non-catalytic alpha-beta hydrolases. $\mathrm{J}$ Mol Biol, 2004, Vol. 2: 519-30.

9. Wilkinson RJ, KA Wilkinson, KA De Semet, et al. Human T and B cell reactivity to the $16 \mathrm{kDa}$ alpha-crystallin protein of Mycobacterium tuberculosis. Scand J Immunol, 1998, Vol. 48: 403-409.

10. Rabahi, MF, Junqueira Kipnis AP, Guerreiro Reis MC, OelemanN W, Conde MB. Humoral response to HSPX and GLcB to previous and recent infection by Mycobacterium tuberculosis. BMC Infect Dis, 2007, Vol. 7: 148-56. 
11. Almeida CMC, Vasconcelos Jr AC, Kipnis A, Andrade AL and Junqueira Kipnis AP. Humoral immune responses of tuberculosis patients in Brazil indicate recognition of Mycobacterium tuberculosis MPT51 and GLcB. Clin Vaccine Immunol, 2008, Vol. 15: 579-81.

12. Laal S, Samanich KM, Sonnenberg MG, et al. Human humoral immune responses to antigens of Mycobacterium tuberculosis: immunodominance of high-molecular-mass antigens. Clin Diagn Lab Immunol, 1997, Vol. 4 (1): 49-56.

13. Lyashchenko K, Colangeli R, Dude M, et al. Heterogenous antibody responses in tuberculosis. Infect Immun, 1998, Vol. 66 (8): 3936-40.

14. Mori T, Sakatani M, Yamagishi F, et al. Specific detection of tuberculosis infection. Am J Respir Crit Care Med, 2004, Vol. 170: 59-64.

15. Singh KK, Dong Y, Belisle JT, et al. Antigens of Mycobacterium tuberculosis recognized by antibodies during incipient, subclinical tuberculosis. Clin Diagn Lab Immunol, 2005, Vol. 12 (2): 35458.

16. Mirtskhulava V, Kempker R, Shields KL et al. Prevalence and risk factors for latent tuberculosis infection among health care workers in Georgia. Int J Tuberc Lung Dis, 2008, Vol. 12 (5): 513-19.

17. Lopes LK, Teles SA, Souza AC, Rabahi MF, Tiplle AF. Tuberculosis risk among nursing professionals from central Brazil. Am J Infect Control, 2008, Vol.36(2): 148-51.

18. Roth VR, Garret DO, Laserson KF et al. A multicenter evaluation of tuberculin skin test positivity and conversion among health care workers in Brazilian hospitals. Int J Tuberc Lung Dis, 2005, Vol. 9 (12):1335-42.

19. Menzies D, Joshi R and Pai M. Risk of tberculosis infection and disease associated with work in health care setting. Inter $\mathrm{J}$ Tuberc Lung Dis, 2007, Vol. 11(6): 593-605.

20. Joshi R, Reingold AL, Menzies D and Pai M. Tuberculosis among health-care workers in low- and middle-income countries: a systematic review. Plos Med, 2006, Vol. 3(12): 2376-91.

21. Alonso-Echanove J, Granich RM, Lazlo A, et al. Occupational transmission of Mycobacterium tuberculosis to health care workers in a University Hospital in Lima, Peru. Clin Infect Dis, 2001, Vol. 33(5): 589-96.

22. Hussain R, Dawood G, Abrar N, et al. Selective increase in antibody isotypes and immunoglobulin $\mathrm{G}$ subclass responses to secreted antigens in tuberculosis patients and healthy household contacts of the patients. Clin Diagn Lab Immunol, 1995, Vol.2(6): 72632.

23. Mineo JR, Khan IA and Kasper LH. Toxoplasma gondii: a monoclonal antibody that inhibits intracellular replication. Exp Parasitol, 1994, Vol. 79: 351-61.

24. Mukherjee J, G Nussbaum, MD Scharff and A Casadevall. Protective and nonprotective monoclonal antibodies to Cryptococcus neoformans originating from one B cell. J Exp Med, 1995, Vol. 181: 405-09.

25. Chang Z, Primm TP, Jakana J, et al. Mycobacterium tuberculosis 16-kDa antigen (Hsp16.3) functions as an oligomeric structure in vitro to suppress thermal aggregation. J Biol Chem, 1996, Vol. 271(12): 7218-23.

26. Ramalingam B, AR Baulard, C Locht, PR Narayanan and Raja A. Cloning, expression, and purification of the $27 \mathrm{kDa}$ (MPT51, Rv3803c) protein of Mycobacterium tuberculosis. Protein Exp Purif, 2004, Vol. 36(1): 53-60.
27. Pai M, Riley LW and JM Colford Jr. Interferon-gamma assays in the immunodiagnosis of tuberculosis: systematic review. Lancet Infect Dis, 2004, Vol. 4(12): 761-76.

28. Fietta A, Meloni F, Cascina A, et al. Comparison of a whole-blood interferon-gamma assay and tuberculin skin testing in patients with active tuberculosis and individuals at high or low risk of Mycobacterium tuberculosis infection. Am J Infect Control, 2003, Vol. 31: 347-53.

29. Huebner RE, Schein MF, Bass JBJ. The tuberculin skin test. Clin Infect Dis, 1993, Vol. 17: 968-75.

30. Farhat M, Greenaway C, Pai M and Menzies D. False-positive tuberculin skin tests: what is the absolute effect of BCG and non-tuberculous mycobacteria. Int J Tuberc Lung Dis, 2006, Vol. 10: 1192-1204

31. Primm TP, Lucero CA, Falkinham JO 3rd. Health impacts of environmental mycobacteria. Clin Microbiol Rev, 2004, Vol. 17 (1): 98-106.

32. Bardana EJ, JK McClatchy, RS Farr, and P Minden. Universal occurence of antibodies to tubercle bacilli in sera from nontuberculous and tuberculous individuals. Clin Exp Immunol, 1973, Vol. 13: 65-77.

33. Grange, JM. The humoral immune response in tuberculosis: its nature, biological role and diagnostic usefulness. Adv Tuberc Res, 1984, Vol. 21: 1-78.

34. Samanich K, JT Belisle and Laal S. Homogeneity of antibody responses in tuberculosis patients. Infect Immun, 2001, Vol. 69(7): 4600-09.

35. Wanchu A, Dong Y, Sethi S, et al. Biomarkers for clinical and incipient tuberculosis: performance in a TB-endemic country. Plos ONE, 2008, Vol. 3(4): e2071.

36. Achkar JM, Dong Y, Holzman RS, et al. Mycobacterium tuberculosis malate synthase- and MPT51-based serodiagnostic assay as an adjunct to rapid identification ofpulmonary tuberculosis. Clin Vaccine Immunol, 2006, Vol. 13(11): 1291-93.

37. Beck ST, Leite OM, Arruda S, and Ferreira AW. Humoral immune response to low molecular weight antigens of Mycobacterium tuberculosis by tuberculosis patients and contacts. Braz J Med Biol Res, 2005, Vol. 38(4): 587-96.

38. Pinchuk LM, SJ Klaus, DM Magaletti, et al. Functional CD40 ligand (CD40L) expressed by human blood dendritic cells is up-regulated by CD40 ligation. J Immunol, 1996, Vol. 158: 120-26.

39. Kaufmann, SHE. Protection agaisnt tuberculosis: cytokines, $\mathrm{T}$ cells and macrophages. Ann Rheum Dis, 2002, Vol. 61 (Suppl II): 54-58.

40. D'Ombrain MC, Hansen DS, Simpson KM and Schofield L. Gammadelta-T cells expressing NK receptors predominate over NK cells and conventional $\mathrm{T}$ cells in innate IFN-gamma response to Plasmodium falciparum malaria. Eur J Immunol, 2007, Vol. 37(7): 1864-73.

41. Ota MOC, Vekemans J, Schelgel-Haueter SE, et al. Influence of Mycobacterium bovis Calmette-Guérin BCG on antibody and cytokineresponses to human neonatal vaccination. J Immunol, 2002, Vol. 168: 919-25.

42. Nabeshima S, Murata M, Kashiwagi K, et al. Serum antibody response to tuberculosis-associated glycolipid antigen after BCG vaccination in adults. J Infect Chemoter, 2005, Vol. 11(5): 256-58.

43. Drowart A, Selleslaghs J, Yernault JC, et al. The humoral immune response after BCG vaccination: an immunoblotting study using two purified antigens. Tuber Lung Dis, 1992, Vol. 73(3): 137-40. 